\title{
Early Prosthetic Valve Endocarditis
}

National Cancer Institute

\section{Source}

National Cancer Institute. Early Prosthetic Valve Endocarditis. NCI Thesaurus. Code C128357.

Prosthetic valve endocarditis that occurs days to weeks after surgery. 